\title{
Cortisol levels in response to starting school in children at increased risk for social phobia
}

Article

Other

Russ, S. J., Herbert, J., Cooper, P., Gunnar, M. R., Goodyear, I., Croudace, T. and Murray, L. (2012) Cortisol levels in response to starting school in children at increased risk for social phobia. Psychoneuroendocrinology, 37 (4). pp. 462-474. ISSN 1873-3360 doi:

https://doi.org/10.1016/j.psyneuen.2011.07.014 Available at https://centaur.reading.ac.uk/25344/

It is advisable to refer to the publisher's version if you intend to cite from the work. See Guidance on citing.

To link to this article DOI: http://dx.doi.org/10.1016/j.psyneuen.2011.07.014

Publisher: Elsevier

All outputs in CentAUR are protected by Intellectual Property Rights law, including copyright law. Copyright and IPR is retained by the creators or other copyright holders. Terms and conditions for use of this material are defined in the End User Agreement. 


\section{CentAUR}

Central Archive at the University of Reading

Reading's research outputs online 


\section{Elsevier Editorial System(tm) for Psychoneuroendocrinology Manuscript Draft}

Manuscript Number: PNEC-D-11-00057

Title: Cortisol levels in response to starting school in children 'at risk' for social phobia

Article Type: Original Research Paper

Keywords: social phobia; intergenerational transmission; cortisol; child development; school adjustment; social stressor; HPA axis

Corresponding Author: Professor Lynne Murray, $\mathrm{PhD}$

Corresponding Author's Institution: University of Reading

First Author: Stephanie Russ, PhD

Order of Authors: Stephanie Russ, PhD; Joe Herbert, PhD; Peter J Cooper, D.Phil; Megan R Gunnar; Ian Goodyer; Timothy Croudace; Lynne Murray, PhD

Abstract: Background: Research on depression has identified hyperactivity of the HPA axis as a potential contributory factor to the intergenerational transmission of affective symptoms. However, this has not yet been examined in the context of social phobia. The current study compared HPA axis activity in response to a universal social stressor (starting school) in children of 2 groups of women: one with social phobia and one with no history of anxiety (comparison group). To determine specificity of effects of maternal social phobia, a third group of children were also examined whose mothers had generalised anxiety disorder (GAD).

Method: Children provided salivary cortisol samples in the morning, afternoon and at bedtime across 3 time-blocks surrounding the school start: a month before starting school (baseline), the first week at school (stress response), and the end of the first school term (stress recovery). Child behavioural inhibition at 14 months was also assessed to explore the influence of early temperament on later stress responses.

Results: All children displayed an elevation in morning and afternoon cortisol from baseline during the first week at school, which remained elevated until the end of the first term. Children in the social phobia group, however, also displayed an equivalent elevation in bedtime cortisol, which was not observed for comparison children or for children of mothers with GAD. Children in the social phobia group who were classified as 'inhibited' at 14 months displayed significantly higher afternoon cortisol levels overall.

Summary: A persistent stress response to school in the morning and afternoon is typical for all children, but children of mothers with social phobia also display atypical elevations in evening cortisol levels when at school - signalling long-term disruption of the circadian rhythm in HPA axis activity. This is the first study to report HPA axis disruption in children at risk of developing social phobia, and future research should aim to determine whether this represents a pathway for symptom development, taking early temperament into account. 
$16^{\text {th }}$ December, 2010

Dear Dr Kalin

'Cortisol levels in response to starting school in children 'at risk' for social phobia'

We would be most grateful if you would consider our paper for publication in the Journal. We feel our study has a number of strengths: Whereas past studies on the familiality of social phobia have tended to suffer from small and heterogeneous study groups, with cross-sectional designs, our study involved a large and diagnostically homogenous group of mothers with social phobia and their children, and utilized a prospective longitudinal design with multiple assessments of child functioning around the ecologically valid, and normative, social stressor of starting school. Further, our protocol for assessment of HPA axis functioning, involving saliva collections, was strong in that we used multiple sampling days, assessment of basal, stressed and recovery cortisol levels, and consideration of the diurnal rhythm. Our central finding of long-term disruption of the circadian rhythm in HPA axis activity in children of mothers with social phobia vs. non-anxious control mothers, following on the social stressor of starting school, is, to our knowledge, entirely novel, since it is the first study to report HPA axis disruption in children at risk of developing social phobia. It is also of scientific importance in opening up the possibility that such disruption may be involved in the process of intergenerational transmission of social phobia.

We hope you will feel that the study is of sufficient interest to merit its consideration. We note on the Journal website that there is a Special issue on Biological Basis of Mood 
Disorders. If you feel that it would be appropriate for our paper to be considered for this issue and the deadline has not already passed, we would be very happy for it to be processed via this route; we thank you for your attention.

Yours sincerely

Lynne Murray 
Cortisol levels in response to starting school in children 'at risk' for social phobia

Russ, S., [1] Herbert, J., [2] Cooper, P.,[1] Gunnar, M.R., [3] Goodyer, I., [4] Croudace, T. ${ }_{[4]} \&$ Murray L.* [1]

1. Winnicott Research Unit, School of Psychology and Clinical Language Science, University of Reading, UK.

2. Department of Clinical Neuroscience, University of Cambridge, UK.

3. Institute of Child Development, University of Minnesota, Minneapolis, USA.

4. Department of Psychiatry, University of Cambridge, UK.

\footnotetext{
*Correspondence to: Lynne Murray

E mail: lynne.murray @rdg.ac.uk
}

Telephone: 00441183786667

Fax: 00441183786665. 


\begin{abstract}
Background: Research on depression has identified hyperactivity of the HPA axis as a potential contributory factor to the intergenerational transmission of affective symptoms. This has not yet been examined in the context of social phobia. The current study compared HPA axis activity in response to a universal social stressor (starting school) in children of 2 groups of women: one with social phobia and one with no history of anxiety (comparison group). To determine specificity of effects of maternal social phobia, a third group of children were also examined whose mothers had generalised anxiety disorder (GAD).
\end{abstract}

Method: Children provided salivary cortisol samples in the morning, afternoon and at bedtime across 3 time-blocks surrounding the school start: a month before starting school (baseline), the first week at school (stress response), and the end of the first school term (stress recovery). Child behavioural inhibition at 14 months was assessed to explore the influence of early temperament on later stress responses.

Results: All children displayed an elevation in morning and afternoon cortisol from baseline during the first week at school, which remained elevated until the end of the first term. Children in the social phobia group, however, also displayed an equivalent elevation in bedtime cortisol, which was not observed for comparison children or for children of mothers with GAD. Children in the social phobia group who were classified as 'inhibited' at 14 months displayed significantly higher afternoon cortisol levels overall.

Summary: A persistent stress response to school in the morning and afternoon is typical for all children, but children of mothers with social phobia also display atypical elevations in evening cortisol levels when at school - signalling longer-term disruption of the circadian rhythm in HPA axis activity. This is the first study to report HPA axis disruption in children at risk of developing social phobia. , Future research should determine whether this represents a pathway for symptom development, taking early temperament into account. 
Keywords: social phobia, intergenerational transmission, cortisol, child development, school adjustment, social stressor, HPA axis 


\section{Introduction}

Social phobia is a disabling anxiety disorder, characterised by an excessive and persistent fear of scrutiny and negative evaluation during social or performance situations. The feared situation will either be endured with dread or avoided completely, resulting in marked restrictions on social functioning. Social phobia is one of the most common psychiatric disorders. It has lifetime and 12 month prevalence rates of around $13 \%$ and 6\%, respectively (Furmark, 2002), and occurs more frequently in females (Furmark, 2002; Kessler et al., 1994; Kessler et al., 1998). The age of onset is typically during adolescence, and if untreated, it tends to run a chronic course, with around half of those with the disorder reporting a life-long history (e.g. Grant et al., 2005; Schneier, et al., 1992; Verhulstet al., 1997). Precursors of the disorder are commonly seen before adolescence, and they can have a profound deleterious impact on child socio-emotional development (Beidel et al., 1999).

As with other affective disorders, social phobia aggregates in families, and clinical studies demonstrate specificity of transmission (i.e. first degree relatives of individuals with social phobia are at increased risk of social phobia, but not other anxiety disorders) (e.g. Fyer et al., 1995; Maier et al., 1993; Merikangas et al., 2003; Stein et al., 1998). A genetic contribution to symptom development has been identified but is modest, with an estimated heritability of around 10\% (Nelson et al., 2000). This implies that additional mechanisms are important in the transmission of symptoms and it appears that both child temperament and parenting style also play important aetiological roles (see review by Murray et al., 2009).

Importantly, a recent strand of research in the context of depression suggests that the involvement of physiological systems, particularly those implicated in the response to stress, such as the Hypothalamic Pituitary Adrenal (HPA) Axis, should also be taken into account when considering the transmission of affective disorders. The HPA axis is an essential part of the organism's homeostatic system, and one of the most reactive of all neuroendocrine systems. The mechanism 
through which the axis operates is characterised by the activation of a cascade of biochemical events, which begins with the release of corticotropin-releasing hormone $(\mathrm{CRH})$ from the hypothalamus, and culminates with the release of glucocorticoids (primarily cortisol) from the adrenal gland (Antoni, 1986; Plotsky, 1991). HPA axis activity is one of the many bodily functions to display a distinct daily or diurnal rhythm. Cortisol levels are at their highest at awakening and then decline over the day, reaching near zero in the evening and remaining low until the later hours of sleep, when levels begin to increase (Clow et al., 2004). Imposed on this rhythm, there is a response to awakening, consisting of a 50-160\% increase in the first 30-40 minutes after eyes open, termed the cortisol awakening response (CAR) (Fries et al., 2009). Inputs to the hypothalamus from forebrain structures appear to be involved in producing the CAR, which fluctuates in anticipation of the stressors or challenges of the day.

While the release of glucocorticoids is critical for enabling adaptation to perturbations in homeostasis caused by both real and anticipated increases in demand, i.e., stress (Selye, 1973), chronically, or frequently elevated cortisol levels (e.g. raised baseline levels or poorly regulated stress responses) are associated with adverse effects on health, including the development of mental health problems, particularly affective disorders (Goodyer et al., 2010; Goodyer et al,. 2009; Goodyer et al., 2003; Goodyer et al., 2001; Harris et al., 2000). A proportion of depressed individuals are reliably reported to display hyperactivity of the HPA axis, as demonstrated by elevated baseline cortisol levels (particularly in the evening), hypertrophy of the adrenal gland, raised cerebral-spinal levels of CRH, and reduced negative feedback in response to administration of synthetic glucocorticoids, such as dexamethasone (Arato et al., 1989; Carroll et al., 2007; Heuser et al., 1994; Rubin et al., 1995). Similarly, elevated basal cortisol levels have been consistently reported in individuals with panic disorder (e.g. Wedekind et al., 2000), and, to a lesser extent, in individuals with OCD (Gustafsson et al., 2008) and GAD (e.g. Mantella et al., 2008). Research in the context of social phobia, however, is more limited. While some studies have reported elevated cortisol levels in response to stress in individuals with social phobia compared to controls, findings have not been consistent (Condren et al., 2002; Furlan et al., 2001; Roelofs et al., 2008; Uhde et al., 1994). 
There is more data on HPA axis functioning in relation to child temperamental traits thought to be associated with the development of social phobia, i.e., behavioural inhibition or BI (Kagan, 1994), Inhibited/socially wary infants have been shown to display the highest cortisol levels under both stressed (e.g. Kagan et al., 1987) and non-stressed (e.g. Schmidt et al., 1997) conditions. Studies involving children undergoing normative stressors, such as entering novel peer-group settings (e.g. starting school), have been particularly fruitful in demonstrating these cortisol-temperament relationships (e.g. Bruce et al., 2002; Gunnar et al., 1997; Tout et al., 1998; Turner-Cobb et al., 2008; Watamura et al., 2003). While such social events are generally considered positive for children, in terms of stimulating the development of social capacities, the social and emotional resources required for their navigation are considerable, and may cause significant stress. It appears that an initial stress response (i.e. rise in cortisol levels) in the morning and afternoon, but not in the evening, is normative for children when entering these settings, and tends to reduce as the environment becomes familiar (e.g. Sumner et al., 2010; Turner-Cobb et al., 2008). However, a number of studies have shown that children with internalising temperamental traits (e.g. teacher rated shyness) are particularly prone to mounting persistent stress responses in these settings, i.e. displaying continued elevations in cortisol when the surroundings are no longer unfamiliar (e.g. Gunnar et al., 1997). Others report that elevations in evening cortisol levels, in particular, are observed for shy children on school days as compared to weekend days; more extroverted children, by contrast, may show elevations in morning cortisol levels that drop to the equivalent of baseline levels by the end of the day (Bruce et al., 2002; Turner-Cobb et al., 2008). Interestingly, where apparently conflicting results have been observed, i.e. with the highest cortisol levels being reported for the most extroverted children, these have been explained by the type of interactions these children display (i.e. with higher levels of bold, risk-taking, problematic behaviour), and if these social interactions are taken into account, the relationship between shyness and high cortisol is upheld (Gunnar et al., 2003).

Two studies have reported a predictive association between elevated cortisol levels and the development of social anxiety. Smider and colleagues (2002) measured children's average afternoon 
cortisol levels at 4.5 years, and found higher levels to be predictive of teacher reported withdrawal (including social wariness) a year and a half later. The same relationship was found using maternal ratings of withdrawal, but for girls only. Importantly, these predictive effects held even when controlling for behaviour assessed at the time the cortisol samples were provided (Smider et al., 2002). In the second study, greater increases in cortisol levels across an 18-month period predicted greater general and social anxiety in 9-14 year old girls (Schiefelbein \& Susman, 2006).

If HPA axis hyperactivity can contribute to the development of affective psychopathology, it could well be important in the intergenerational transmission of such disorders (including social phobia) from mother to child. To substantiate this notion, however, systematic evidence supporting an association between maternal psychopathology and child HPA axis hyperactivity is required. Ashman and colleagues found that 7 year old children of depressed mothers, who demonstrated internalising symptoms themselves, displayed higher basal cortisol levels than children of non-depressed controls; importantly, maternal depression in the child's first 2 years was the most significant predictor of these elevations (Ashman et al., 2002). Similarly, in a study investigating the effects of maternal stress on children, maternal depression in infancy was identified as the strongest predictor of future increased cortisol levels in preschoolers also exposed to concurrent maternal depression (Essex et al., 2002). In another study, 13-year olds exposed to postnatal depression had higher and more variable 8 AM cortisol levels than non-exposed adolescents, and this relationship held when controlling for concurrent child and maternal symptoms, as well as other potential confounds (Halligan et al., 2004). Notably, when directly observed mother-child interactions were examined, maternal withdrawal in infancy (but not at five years) predicted the 13 year morning cortisol elevations (Murray et al., 2010). In turn, these higher morning cortisol levels mediated the effects of maternal postnatal depression on offspring depressive symptoms at age 16, even when controlling for depression at 13 years (Halligan et al., 2007), supporting the idea that HPA axis hyperactivity constitutes a potential process through which the transmission of familial risk for depression occurs. 
Comparable intergenerational studies in the context of maternal anxiety disorders are less numerous, and, indeed, absent in the case of social phobia. Nonetheless, one study (Brennan et al., 2008) did take into account maternal co-morbid anxiety disorders whilst examining the link between child HPA axis activity and maternal depression, and found that, whereas maternal depression was associated with increased offspring baseline mean cortisol levels, depression with co-morbid anxiety was more strongly associated with increased child cortisol reactivity to stress. This suggests the effects of the two disorders on offspring HPA axis activity may not be equivalent, with maternal anxiety showing stronger associations with child elevated responses to stress, rather than the baseline effects commonly observed in depression. This is consistent with the finding that 14-15 month old children of mothers with panic disorder demonstrated elevated cortisol levels in a stressful laboratory setting, but not at home (Warren et al., 2003).

Overall the evidence supports an association between HPA axis hyperactivity and the development of affective disorders (including social anxiety), and suggests that maternal affective disorder has a putative causal association with offspring HPA axis hyperactivity. This indicates that there may be intergenerational transmission of social phobia mediated in part by dysregulated cortisol activity. To our knowledge, no studies have yet attempted to characterise HPA axis activity in a group of off-spring at risk for social phobia by virtue of a maternal diagnosis of this disorder. The aim of the current study was to characterise HPA axis activity in response to the social stressor of starting school in 4-5 year-old children of mothers with social phobia and a similarly aged comparison group of children of mothers with no history of anxiety. We tested the hypothesis that offspring of social phobic mothers would demonstrate cortisol hyperactivity compared to offspring of mothers with no lifetime history of anxiety disorders. Secondly, we tested for specificity effects by comparing offspring of mothers with social phobia with those of mothers with general anxiety disorder. This was done in the context of a wider ongoing longitudinal study on the intergenerational transmission of social phobia, as part of which child BI had been assessed at 14 months (Murray et al., 2007; 2008). In the current study, the mothers and their children, then aged 4-5 years, were reassessed (i) one month before 
starting school (BSS) (ii) across the first week at school (FWS), and (iii) at the end of the first school term (EoT), with salivary cortisol levels sampled over several days, across the course of the day, for each of these three time blocks. Starting school is an ideal setting for assessing individual differences in HPA axis activity in response to social challenge, being a normative, yet socially demanding, event. Based on previous findings (e.g. Gunnar et al., 1997), we expected all children to show some initial cortisol response, i.e. an elevation in cortisol levels, but that this would be more pronounced and persistent (i.e. extending to the end of the first term) for those in the social phobia group, thus indicating poorer habituation to the social stressor. Furthermore, we expected, on the basis of previous findings (Bruce et al., 2002), that this would apply particularly to evening/bedtime cortisol levels. When investigating these effects, it was considered important to take child sex and behavioural inhibition into account, given that both have been shown to affect cortisol (Kagan et al., 1987; Rosmalen- et al., 2005).

In addition, given that an important recent issue to emerge in the anxiety disorder literature is the notion of specificity of transmission and the idea that different processes might be involved in the intergenerational transmission of different anxiety disorders (Murray et al., 2009), as a final step we included in our study a further anxious group, namely, children whose mothers had generalised anxiety disorder (GAD). This enabled us to determine whether any differences observed between children of mothers with social phobia and children of comparison mothers were specific to a maternal diagnosis of social phobia, rather than a feature of maternal anxiety more generally.

\section{Method}

\section{Sample Recruitment.}

Women were screened during pregnancy by questionnaire for Social Phobia and GAD at routine 20-week antenatal clinics using items from the Social Interaction and Anxiety Scale- SIAS, 
and the Social Phobia Scale - SPS, and from the Penn State Worry Questionnaire - PSWQ (Mattick \& Clark, 1998; Meyer et al., 1990). Probable cases were systematically interviewed to confirm diagnosis; and a random sample of probable non-cases was similarly interviewed to check that they had neither disorder, for inclusion in the comparison group ${ }^{1}$. Recruitment was confirmed after delivery, providing the infant was healthy (e.g. not premature, no major congenital disorder). Final numbers were: Social Phobia (but not GAD) $n=67$, GAD (but not Social Phobia) $n=56$, and comparison group (no anxiety) $\mathrm{n}=94$. Mothers and their infants were assessed through the first two years postpartum, including an assessment of infant BI at 14 months (see Murray et al., 2008); families were then re-contacted when the children were aged four years. Numbers participating in the four-year follow-up were: Social Phobia $n=53 ;$ GAD $n=44 ;$ Comparison $n=67$. Those with complete data for the currently reported cortisol assessments are as follows: Social Phobia $n=36$; GAD $n=30 ;$ Comparison $n=46$.

The three groups did not differ on demographic measures (maternal age, marital status, education, social class, sex of child), and nor did these participants differ from those who were not followed up, except in one respect: the mothers of those not followed up were somewhat younger than those assessed $($ mean $=29.45(\mathrm{SD}=4.81)$ yrs, vs. mean $=31.12(\mathrm{SD}=3.99)$ yrs, respectively; $\mathrm{t}=-2.276$, $p<.05)$. Sample characteristics for the current assessment are shown in Table 1.

Table 1 about here

\section{Measures}

\section{Maternal mental state}

Mothers were interviewed at recruitment and at follow up by trained psychologists and mental health clinicians using the Structured Clinical Interview for DSM-IV Axis 1 disorders (SCID-1; First et al., 1995). All interviews were subsequently discussed with a senior clinical team to confirm diagnosis and, at recruitment, allocation to the three study groups. At five years, some mothers in each

\footnotetext{
${ }^{1}$ For full details of recruitment procedures, see Murray et al., 2007.
} 
anxiety group no longer met full criteria for a DSM-IV diagnosis (23 (46\%) in the Social Phobia group and $16(42 \%)$ in the GAD group). Nevertheless, the two index groups' symptoms still differed substantially from those of the comparison mothers on the relevant symptom scales, as well as differing from each other (all pairwise comparisons $p<.01$; see Table 1).

\section{Child Behavioural Inhibition at 14 months.}

This was assessed according to the paradigm devised by Kagan and colleagues (1987), in which latencies to approach, and fearful or distressed behaviours towards novel non-social and social stimuli, are assessed. In the current study we scored seven observation frames for infant responses to: a mechanical dinosaur that moved and 'roared' (three one-minute observations); a series of novel toys in an unfamiliar playroom (one continuous three minute observation); and an approach by an unfamiliar female stranger (three observations- approach, pick up, and play phases). Infant behavioural inhibition was scored as present or absent in each of the seven periods, and the resultant score (0-7) made into a binary variable (>3) defining the $27.5 \%$ most inhibited infants.

\section{Cortisol}

Cortisol was assessed via saliva samples. Mothers were asked to collect saliva samples from their child during three time-blocks around the school start: one month before starting school (BSS - 3 consecutive days); the first week at school (FWS- 5 consecutive days), and near the end of the first term (EoT- mid-way through the second half of the first term - 4 consecutive days). BSS samples were collected on days that were considered by the mother to be typical for the child, avoiding any exciting trips or events, any 'taster' days at the school, and also child illness. Samples collected in the second two blocks were collected only on days that the child attended school. For the FWS block, if weekend days interrupted sample collection (i.e. if the child started school on any day other than Monday), nonschool (weekend) days were skipped, and collection resumed the following Monday. EoT samples were initiated on a Monday or Tuesday to avoid weekends. To assess the degree of reduction in 
cortisol levels across the day, three samples were provided on each sampling day: one within 30 minutes of waking (morning), one at 4pm (afternoon), and one within 30 minutes of bedtime (bedtime). Thus, with 12 days of sampling (3 BSS, 5 FWS, and 4 EoT) and 3 samples provided per day, a maximum of 36 cortisol samples were provided by each child.

Children provided saliva by spitting into $12.5 \mathrm{ml}$ vials. If any child had particular difficulty producing saliva, parents were provided with Trident Original sugarless gum, which is effective at stimulating saliva flow and has been shown to have a negligible effect on cortisol levels (Schwartz et al., 1998). Fifty one percent of children providing samples used gum (of these, only $7 \%$ required it for all samples). Children rinsed their mouth with water 5 minutes before providing a sample (to avoid sample dilution), and the procedure was carried out before meals and before brushing teeth. It was requested that sampling time and waking time (for morning samples) be recorded each time. Cortisol levels were determined using an enzyme linked immunoadsorbant assay (ELISA) technique (Cooper et al., 1989). The inter- and intra-assay coefficients of variation were $9.90 \%$ and $4.47 \%$ respectively.

\section{Data Management}

\section{Compliance}

Of those providing samples, $79.6 \%$ completed the full-set: $84.3 \%$ of the BSS samples, $80 \%$ of the FWS samples and $75.7 \%$ of the EoT samples ${ }^{2}$. Cortisol levels were available for $88 \%$ samples provided: $88.3 \%$ for BSS, $86.4 \%$ for FWS, and $91.4 \%$ of the EoT. Waking time was recorded for $63.4 \%$ of the BSS samples, $67.9 \%$ of the FWS samples and $64.3 \%$ of the EoT samples. There was no significant difference between study groups in the number of samples provided, the number of samples for which cortisol levels were available, or the number of morning samples for which waking time was provided (all p-values .>0.05, range-.34-.99).

\footnotetext{
${ }^{2}$ For a small number of individuals, cortisol levels could not be determined for some of the samples provided, due to insufficient amounts of saliva for assaying.
} 


\section{Data preparation}

Cortisol levels more than 2.5 standard deviations above the overall mean were likely to have been sampled incorrectly or affected by unrecorded medication. As such, they were removed as outliers before computing the final summary statistics. Furthermore, participants were excluded if they did not have data available at all three time-blocks for either their morning, afternoon or bedtimes samples. The final sample numbers included in the main analyses are shown in Table 2. Mean morning, afternoon and bedtime cortisol levels were computed for each of the three time-blocks. If the participant had not provided samples on all days requested, their mean was computed for the reduced number of sampling days attempted. Mean cortisol levels were log-transformed to better approximate normal distributions for parametric statistical analysis.

\section{Data Analyses:}

For mothers in the social phobia and comparison group, mixed 2 (maternal group) x 3 (timeblock) ANOVAs/ANCOVAs were conducted, with repeated measures on the latter factor, for mean morning, afternoon, and bedtime cortisol levels, to determine the pattern of HPA axis activity over time, and any group differences. Where significant effects/interactions were observed, post-hoc tests were carried out with Bonferroni adjustments. Of note, a number of variables were considered that might have affected the data: child age, parity, birth weight, sampling time, waking time (for morning levels only), familiarity with school (attendance at taster day/nursery/preschool), presence of friends at school, number of cortisol samples available and whether or not sampling days were interrupted by a weekend. These variables were included as covariates if: they were directly associated with cortisol levels, or interacted with maternal group or change over time, to predict cortisol levels.

Next, the main effects of sex and BI, and their interactions with maternal group (i.e., their moderating effects), were examined in follow-up analyses. Thus, the initial repeated measures analyses were conducted again but adding in either sex or BI as an additional between-subjects factor. 
Finally, where significant associations between maternal group and cortisol were observed, the same comparisons were made for the comparison and GAD group to see whether the effect was specific to social phobia, or appeared to be associated with more general anxiety.

\section{Results}

Descriptive statistics for the cortisol data are presented in Table 2. As expected, a clear diurnal reduction in cortisol levels across the day can be observed at all time-blocks. In general, mean sampling times displayed reasonably low variation, and were in accordance with the intended protocol. Importantly, morning samples were generally collected within 30 minutes of waking, as requested. Consistent with previous research, however, (e.g. Bruce et al., 2002), both FWS and EoT morning cortisol samples were provided earlier than those in the BSS time block (BSS-FWS: difference $=.36$, $\mathrm{p}<.001$; BSS-EoT: difference=.4, $\mathrm{p}<.001$ ), probably because children were getting up earlier for school. As this might have resulted in higher morning cortisol levels on school days, sampling time was controlled for in analyses wherever it was related to the change in cortisol over time. Afternoon and bedtime samples were provided at equivalent times across the 3 time-blocks (all p-values $>0.05$, range .37-.46). There were no group differences in sampling times or in the time elapsing between waking and providing the morning sample (p-values range .12-.99). Additionally, the within-timeblock, day-to-day stability of morning, afternoon and bedtime cortisol levels was examined. Cronbach's alpha suggested a moderate level of day to day stability (internal consistency reliability) in cortisol overall (Cronbach range -.2 -.66, mean=.51), indicating that the use of mean cortisol levels in analyses was appropriate.

A main effect of time-block was observed for morning $(\mathrm{F}(2,94)=5.161, \mathrm{p}=.008)$, afternoon $(\mathrm{F}$ $(2,108)=.001, \mathrm{p}=.001)$ and bedtime cortisol levels $(\mathrm{F}(2,104)=6.108, \mathrm{p}=.003)$. These effects are displayed in Figures 1-3 for children in the comparison and social phobia group separately; along with 
mention of any variables that were included as covariates in the analyses (i.e. adjusted means are shown).

For morning cortisol, a similar pattern of results was observed for children in the comparison and social phobia group. In both, cortisol levels increased significantly from BSS to FWS (mean $\operatorname{diff}=.065, \mathrm{p}=.05$ ), and failed to subside significantly by the EoT time-block (mean diff=.001, $\mathrm{p}=.99$ ).

Similarly, for afternoon cortisol, while none of the individual pair-wise comparisons between time-blocks was significant, the main effect of time seems to reflect an elevation in afternoon cortisol levels from BSS towards EoT for both the comparison group and the social phobia group.

In contrast, for bedtime cortisol levels, the main effect of time can be seen to be driven by the social phobia group alone, as indicated by a significant time-block $\mathrm{x}$ status interaction $(\mathrm{F}(2,104)=$ 4.001, $\mathrm{p}=.021$ ). Examination of effects within each group showed no simple main effect of time-block for the comparison group $(\mathrm{F}(2,51)=.02, \mathrm{p}=.98)$, whereas there was such an effect for the social phobia group $(\mathrm{F}(2,51)=8.501, \mathrm{p}=.001)$, with the FWS and EoT cortisol levels being significantly higher than the Before school levels (BSS-FWS difference $=.223, \mathrm{p}=.004$ and BSS-EoT difference $=.266, \mathrm{p}=.002$ ), but not significantly different from each other (FWS-EoT difference=.04, $\mathrm{p}=.99$ ). Thus, while the children in the comparison group displayed no evident HPA response to school in their bedtime levels, children in the social phobia group displayed an elevation during the first five days of school which did not subside by the end of term. Notably, although children in the social phobia group appear to display lower baseline (BSS) bedtime cortisol levels than those in the comparison group, this difference was not significant $(\mathrm{t}(63)=1.39, \mathrm{p}=.10)$. Furthermore, even when controlling for cortisol levels before the start of school, those at FWS were still slightly higher for children in the social phobia group than those in the comparison group $(\mathrm{F}(1,59)=2.809, \mathrm{p}=.09)$. (Although this was not found at the EoT time block $(\mathrm{F}(1,54)=2.331, \mathrm{p}=.13)$. Together, this suggests that although the initial interaction may, to some extent, have been driven by baseline differences, children in the social phobia 
group did demonstrate a meaningful elevation in bedtime cortisol, to levels exceeding that of children in the comparison group, at least at the FWS time-block.

Of note, child birth order (i.e. whether the study child was the first-born or not), interacted with the change in cortisol over time for morning, afternoon and bedtime cortisol levels and so this variable was included as a covariate in each of the original corresponding analyses (see Figures 1-3). For morning and bedtime cortisol levels there was an interaction between birth order and time (morning: $\mathrm{F}$ $(2,122)=3.707, \mathrm{p}=.03$; bedtime: $\mathrm{F}(2,102)=3.263, \mathrm{p}=.047)$, whereby first-born children displayed a significant elevation in cortisol levels over time (morning: $F(2,60)=2.904, p=.06$; bedtime: $F(2,50)=$ 5.893, $\mathrm{p}=.005$ ) but children with older siblings did not (p-values range: .13-.14). For afternoon cortisol levels, this effect was confined to the social phobia group, which was reflected by a 3-way parity $\mathrm{x}$ maternal status $\mathrm{x}$ time interaction $(\mathrm{F}(2,110)=3.419, \mathrm{p}=.036)$, but again, those who were first born displayed a significant elevation in cortisol levels over time $(F(2,23)=8.389, p<0.01)$, whereas those with older siblings showed only a trend for this to occur $(F(2,23)=2.847, p=.08)$.

Next, the impact of sex and BI (a categorical variable representing the binary grouping BI versus not) was assessed independently by adding each as a factor into the original morning, afternoon and bedtime repeated measures analyses. Where sex was concerned, an interaction between time and sex for morning cortisol was observed $(\mathrm{F}(2,90))=3.397, \mathrm{p}=.04)$, whereby boys displayed a simple main effect of time $(\mathrm{F}(2,44)=5.58, \mathrm{p}=.01)$ but girls did not $(\mathrm{F}(2,44)=.108, \mathrm{p}=.90)$. That is, cortisol levels increased significantly for boys between before school and FWS (mean diff $=.136, \mathrm{p}=.01$ ) and remained high until the end of term (mean diff $=.02, \mathrm{p}=.99$ ), whereas girls showed fairly stable morning cortisol levels across the 3 time-blocks (see Figure 4). No additional relationships between sex and cortisol were observed. Were BI was concerned, there was some evidence for an interaction between maternal status and child BI for afternoon cortisol $(\mathrm{F}(1,48)=3.529, \mathrm{p}=.066)$. Thus, children in the social phobia group who had been classified as highly inhibited at 14 months showed the highest afternoon cortisol levels, significantly higher than those children in the social phobia group who had 
been classified as uninhibited $(\mathrm{F}(1,48)=5.78, \mathrm{p}=.02)$. In contrast, for the comparison group, there was no difference in afternoon cortisol levels between children previously classified as inhibited and uninhibited (See Figure 5).

Finally, to test the specificity of the group effect for bedtime cortisol to social phobia, the equivalent analyses were run for the comparison and GAD groups. No relationships between group status and cortisol were observed, for morning, afternoon or bedtime levels (p-values range .18-.82). Additionally, the main effect of time was upheld for morning cortisol levels (although only marginally $(\mathrm{F}(2,110)=2.486, \mathrm{p}=.088$ and afternoon cortisol levels $(\mathrm{F}(2,96)=5.58, \mathrm{p}=.005)$, but not bedtime cortisol levels $(\mathrm{F}(2,96)=.249, \mathrm{p}=.78)$.

\section{Discussion}

There have been no previous examinations of HPA axis activity in children at risk for social phobia, despite previous research in the context of depression and anxiety suggesting that a hyperactive profile of cortisol activity might be implicated in the intergenerational transmission of affective disorders. To address this issue, the current study compared cortisol levels between children of mothers with social phobia and a comparison group of children of mothers with no history of anxiety disorder, in response to a normative social stressor - starting school. Specifically, the primary aim was to examine whether there were any group differences in cortisol reactivity and recovery in response to starting school. Based on the limited previous work carried out in this area (e.g. Bruce et al., 2002; Gunnar et al., 1997), we expected that all children would show some cortisol stress response to starting school, but that children of mothers with social phobia might show less habituation/recovery, particularly for bedtime measures.

In line with these predictions, morning and afternoon cortisol levels revealed evidence for a universal stress response. Average morning cortisol levels increased significantly during the first five 
days at school, compared to measures taken one month before (baseline, BSS), and remained elevated until the end of term. Similarly, average afternoon cortisol levels were highest at the end of term, although this did not represent a significant elevation over baseline levels. Notably, the finding that morning and afternoon cortisol levels remained elevated to some extent at the end of term for all children is in some contrast to previous studies (e.g. Turner-Cobb et al., 2008), where cortisol levels have been found to be comparable to baseline after a number of weeks at school. Thus, the current findings imply that some degree of cortisol response is mounted on all school days compared to nonschool days - in line with a repeated preparatory/reactive stress response, perhaps serving to equip the child for coping with the continual demands inherent in the school/peer environment.

The pattern of results for bedtime cortisol was different. While children in the comparison group did not show any evidence of an increase in bedtime cortisol on starting school, those in the social phobia group displayed a significant elevation in bedtime cortisol, which remained elevated over the BSS baseline, at the end of term. This effect was likely to have resulted in part from the fact that children in the social phobia group had somewhat, albeit not significantly, lower baseline bedtime cortisol levels than comparison children. Nevertheless, for two reasons this should not be seen to negate the importance of the overall findings: First, when controlling for baseline differences, there was still a trend for children of mothers with social phobia to have higher bedtime cortisol levels than those in the comparison group on the first five days at school (albeit not the end of term). Second, it can be argued that the pattern of cortisol levels over time is actually more informative regarding functional and dysfunctional profiles of HPA axis activity than are actual cortisol levels at any one time, as this allows identification of perturbations in individuals' normative physiological condition.

Taken together, these findings suggest that, despite showing elevated morning and afternoon cortisol levels across the school term, the HPA system of children in the comparison group was sufficiently well-regulated as to bring their cortisol back down to near zero levels by bedtime, as at baseline, in accordance with the normative pattern of cortisol activity reported in previous research in 
these settings (Sumner et al., 2010). For children in the social phobia group, by contrast, bedtime cortisol did not descend to basal levels to the same extent when at school, thereby signalling poorer regulation of the stress response, long-term disruption of the circadian rhythm, and a more persistent profile of stress reactivity. Furthermore, this pattern of bedtime cortisol was not observed in children of mothers with GAD, suggesting that it is not a characteristic associated with having an anxious mother in general, but rather is specifically related to having a socially anxious mother.

The results of the current study support our main hypothesis concerning group differences, and are consistent with the reported relationship between cortisol and temperament in other school studies, such as that reported by Bruce and colleagues (2002), and provide some initial avenues for understanding potential moderation effects. Bruce et al found that more surgent/outgoing children displayed elevated morning, but low bedtime, cortisol at school, like the comparison group here, whereas shy children displayed elevated bedtime cortisol levels when at school in comparison to weekend/basal levels, like the social phobia group here. Our findings are also consistent with the pattern of cortisol previously reported in the context of social phobia and other affective disorders in general, i.e. elevated bedtime cortisol and greater reactivity/poorer regulation to stress (e.g. Furlan et al., 2001; Gunnar et al., 1997; Roelofs et al., 2008).

Interestingly, child birth order (i.e. whether first-born or not) was found to influence the physiological response to starting school. Thus, for morning, afternoon and evening cortisol levels, children who were first-born displayed a significant elevation in cortisol levels over time, but those who had an older sibling did not (note that this was only true for the social phobia group where afternoon cortisol levels were concerned). This implies that factors associated with have an older sibling (e.g. familiarity with the school routine, or actually having an older sibling at the same school to provide support) may be sufficient to buffer the stress response to starting school. This should be taken into account in future research. 
In addition to the relationship between bedtime cortisol and maternal group, both sex and BI were found to be related to cortisol levels. First, an interaction between sex and time-block was observed for morning cortisol levels, whereby boys displayed an increase in cortisol in response to starting school which persisted until the end of term, whereas girls displayed very little response to starting school. This finding is novel and interesting, suggesting that boys may be more prone to mounting anticipatory stress responses to the school day, whereas boys and girls are equally likely to mount reactive responses (i.e. increases in afternoon cortisol levels). Explanations for this should be explored further. Importantly, however, sex was not related to any of the bedtime cortisol measures, suggesting that girls and boys of socially phobic mothers were equally likely to display the observed profiles of heightened bedtime stress reactivity. Finally, with regard to BI, average afternoon cortisol levels were found to be significantly higher overall in children previously classified as being behaviourally inhibited than those classified as uninhibited, but only if their mother had social phobia. This provides some support for previous results showing increased baseline/stress induced cortisol levels in the context of childhood behavioural inhibition (e.g. Kagan et al., 1987), but goes further in suggesting that certain factors associated with having a socially anxious mother drive this relationship. Again, this will be an interesting question to pursue in future work.

In summary, the current study supports previous evidence for a universal stress response in morning and afternoon cortisol levels to starting school which persists until the end of term, in line with a repeated anticipatory/reactive response to the school day. Furthermore, the current study expands on previous work suggesting that boys (for morning cortisol levels only) and first-born children may be particularly at risk of mounting prolonged stress responses during this transition. In relation to our primary aim, and of central interest to the anxiety literature, this study also provides the first evidence to show a link between maternal social phobia and elevations in offspring HPA axis activity to social stress (which is not observed in children of mothers with GAD). Specifically, children of socially phobic mothers were shown to demonstrate elevations in bedtime cortisol levels over baseline across the entire first school term, whereas in children of comparison mothers cortisol 
levels went down on school days, to levels comparable to those observed at baseline. This signals long-term disruption of the circadian rhythm and a potentially dysfunctional profile of stress reactivity in index group children. Additionally, an early temperamental profile of inhibited behaviour was found to be related to higher overall afternoon cortisol levels for children of socially phobic mothers only, suggesting that factors associated with having a socially phobic mother may interact with child temperament to cause elevations in stress hormones. Given the previous findings linking elevated cortisol to the development of affective disorders, including social anxiety (e.g. Goodyer et al., 2010 Smider et al., 2002), these combined findings have notable implications for research on the intergenerational transmission of social phobia. Future work should aim to address the issue of whether persistent elevations in bedtime cortisol might mediate the development of social anxiety in 'at risk' groups. It will also be important to investigate factors that might explain the cortisol elevations observed in 'at risk' children; for example, recent work has suggested that certain 'insensitive' styles of parenting might programme poorer regulation or greater sensitivity of the HPA axis to stress. DeCaro and Worthman (2008), for example, found an association between both low maternal warmth and highly dysfunctional mother-child relationships, and elevated evening cortisol in children during their first term at school.

The current study had a number of methodological strengths, particularly with regard to the sample. Thus, whereas past studies on the familiality of social phobia have tended to suffer from small and heterogeneous study groups, with cross-sectional designs, this study involved large and diagnostically homogenous study groups, and utilised a prospective longitudinal design with multiple assessment points, enabling the systematic assessment of the predictive role of potential aetiological risk factors, such as BI, rather than simply reporting on group differences at a single time-point. Although probably under-powered for anything other than moderate interactions, some evidence for a priori moderation hypotheses was also found. Additionally, the protocol for saliva collections was strong in that it took into account several recommendations made in previous research (i.e. multiple sampling days, the assessment of basal, stressed and recovery cortisol levels, and consideration of the 
diurnal rhythm). Despite these strengths, it is important to note some limitations to the study. First, although the cortisol sampling technique was managed by most participants, whereby the children were required to spit into small pots, it did prove difficult for some children, even with the aid of gum. A few mothers commented that they would have provided more samples if the procedure had not been so time consuming and difficult for their child. Given this, alternative methods might have been desirable, for example, using a straw to transfer the saliva into the vial (e.g. Bruce et al., 2002), or there are a number of other highly absorbent devices currently available (e.g. Groschl et al., 2008). Second, by sampling within the first 30 minutes following wakeup, our early morning samples reflected both the diurnal rhythm and the CAR. From a scientific standpoint, it would have been preferable to take an eyes-open and then 30 minute sample to disentangle the diurnal and CAR components of early morning HPA activity. From a practical standpoint, however, it was felt that the participant burden would be too great if we required parents to take a sample the moment the child awoke and then accurately time a wake+30 minute sample.

While it is evident from the current results that children in the social phobia group displayed persistent bedtime cortisol elevations at school, it is not clear whether or not these elevations, at the end of term for example, would also have been seen under non-stressed conditions i.e. whether the transition into school had caused lasting alterations in more general HPA functioning. It would have been useful, therefore, to have taken a follow-up basal measure of cortisol (i.e. out of school hours, perhaps in the holidays), to assess whether the elevations in bedtime cortisol levels for the social phobia group remained high, or returned to the former baseline levels. It might be expected that, once out of the school setting, levels should return to baseline (as long as no other stress is imposed). However, if there were evidence to support long term alterations in HPA activity this would be of particular concern considering the implications chronically elevated cortisol have for future physical and mental health. 


\section{Acknowledgements}

The research was supported by a grant from the MRC (UK) to Lynne Murray and Peter Cooper, and by a studentship award to Stephanie Russ from the ESRC (UK). We thank Adriane Arteche for statistical advice. 


\section{References}

Antoni, F.A. (1986). Hypothalamic Control of Adrenocorticotropin Secretion: Advances since the Discovery of 41-Residue Corticotropin-Releasing Factor. Endocrine Reviews 7 (4): 351-378.

Arato, M., Banki, C.M., Bissette, G. \& Nemeroff, C.B. (1989). Elevated CSF CRF in suicide victims. Biological Psychiatry 25 (3): 355-359.

Ashman, S.B., Dawson, G., Panagiotides, H., Yamada, E. \& Wilkinson, C.W. (2002). Stress hormone levels of children of depressed mothers. Development and Psychopathology 14 (2): 333-349.

Beidel, D.C., Turner, S.M. \& Morris, T.L. (1999). Psychopathology of childhood social phobia. Journal of the American Academy of Child and Adolescent Psychiatry 38 (6): 643-650.

Brennan, P.A., Pargas, R., Walker, E.F., Green, P., Newport, J.D. \& Stowe, Z. (2008). Maternal depression and infant cortisol: influences of timing, comorbidity and treatment. Journal of Child Psychology and Psychiatry 49 (10): 1099-1107.

Bruce, J., Davis, E.P. \& Gunnar, M.R. (2002). Individual differences in children's cortisol response to the beginning of a new school year. Psychoneuroendocrinology 27 (6): 635-650.

Carroll, B.J., Cassidy, F., Naftolowitz, D., Tatham, N.E., Wilson, W.H., Iranmanesh, A., Liu, P.Y. \& Veldhuis, J.D. (2007). Pathophysiology of hypercortisolism in depression. Acta Psychiatrica Scandinavica 115: 90-103

Clow, A., Thorn, L., Evans, P. \& Hucklebridge, F. (2004). The awakening cortisol response: Methodological issues and significance. Stress - The Intenational Journal on the Biology of Stress 7 (1): 29-37.

Condren, R.M., O’Neil, A., Ryan, M.C.M., Barrett, P. \& Thakore, J.H. (2002). HPA axis response to a psychological stressor in generalised social phobia. Psychoneuroendocrinology 27 (6): 693-703.

Cooper, T.R., Trunkfield, R., Zanella, A.J. \& Booth, W.D. (1989). An enzyme linked immunoadsorbent assay for cortisol in saliva of man and domestic animals. Journal of Endocrinology 123: R13-16.

DeCaro, J.A. \& Worthman, C.M. (2008). Return to school accompanied by changing associations between family ecology and cortisol. Developmental Psychobiology 50 (2): 183-195. 
Essex, M.J., Klein, M.H., Cho, E. \& Kalin, N.H. (2002). Maternal stress beginning in infancy may sensitize children to later stress exposure: Effects on cortisol and behavior. Biological Psychiatry 52 (8): 776-784.

First, M.B., Spitzer, R.L., Gibbon, M. \& Williams, J.B.W. (1995). Structured Clinical Interview for DSM-IV Axis 1 disorders (SCID-1). Washington, DC., American Psychiatric Press.

Fries, E., Dettenborn, L. \& Kirschbaum, C. (2009). The cortisol awakening response (CAR): Facts and future directions. International Journal of Psychophysiology 72 (1): 67-73.

Furlan, P.M., DeMartinis, N., Schweizer, E., Rickels, K. \& Lucki, I. (2001). Abnormal salivary cortisol levels in social phobic patients in response to acute psychological but not physical stress. Biological Psychiatry 50 (4): 254-259.

Furmark, T. (2002). Social Phobia: Overview of community surveys. Acta Psychiatrica Scandinavia 105: 84-93.

Fyer, A.J., Mannuzza, S., Chapman, T.F., Martin, L.Y. \& Klein, D.F. (1995). Specificity in familial aggregation of phobia disorders. Archives of General Psychiatry 52 (7): 564-573.

Goodyer, I.M., Herbert, J. \& Tamplin, A. (2003). Psychoendocrine antecedents of persistent firstepisode major depression in adolescents: a community-based longitudinal enquiry. Psychological Medicine 33 (4): 601-610.

Goodyer IM, Bacon A, Ban M, Croudace T, Herbert J. (2009). Serotonin transporter genotype, morning cortisol and subsequent depression in adolescents. British Journal of Psychiatry. 195 (1):39-45

Goodyer IM, Croudace T, Dudbridge F, Ban M, Herbert J. (2010). Polymorphisms in BDNF (Val66Met) and 5-HTTLPR, morning cortisol and subsequent depression in at-risk adolescents. British Journal of Psychiatry 197:365-71.

Goodyer, I.M., Park, R.J., Netherton, C.M. \& Herbert, J. (2001). Possible role of cortisol and dehydroepiandrosterone in human development and psychopathology. British Journal of Psychiatry 179: 243-249.

Grant, B.F., Hasin, D.S., Blanco, C., Stinson, F.S., Chou, P., Goldstein, R.B., Dawson, D.A., Smith, S., Saha, T.D. \& Huang, B. (2005). The epidemiology of social anxiety disorder in the United States: 
Results from the national epidemiologic survey on alcohol and related conditions. Journal of Clinical Psychiatry 66 (11): 1351-1361.

Groschl, M., Kohler, H., Topf, H., Rupprecht, T. \& Rauh, M. (2008). Evaluation of saliva collection devices for the analysis of steroids, peptides and therapeutic drugs. Journal of Pharmaceutical and Biomedical Analysis 47: 478-486.

Gunnar, M.R., Sebanc, A.M., Tout, K., Donzella, B. \& van Dulmen, M.M.H. (2003). Peer rejection, temperament, and cortisol activity in preschoolers. Developmental Psychobiology 43 (4): 436-358.

Gunnar, M.R., Tout, K., deHann, M., Pierce, S. \& Stansbury, K. (1997). Temperament, social competence, and adrenocortical activity in preschoolers. Developmental Psychobiology 31 (1): $65-$ 85.

Gustafsson, P.E., Gustafsson, P.A., Ivarsson, T. \& Nelson, N. (2008). Diurnal cortisol levels and cortisol response in youths with obsessive-compulsive disorder. Neuropsychobiology 57 (1-2): 1421.

Halligan, S.L., Herbert, J., Goodyer, I.M. \& Murray, L. (2004). Exposure to Postnatal Depression Predicts Elevated Cortisol in Adolescent Offspring. Biological Psychiatry 55: 376-381.

Halligan, S.L., Herbert, J., Goodyer, I. \& Murray, L. (2007). Disturbances in morning cortisol secretion in association with maternal postnatal depression predict subsequent depressive symptomatology in adolescents. Biological Psychiatry 62 (1): 40-46.

Harris, T.O., Borsanyi, S., Messari, S., Stanford, K., Cleary, S.E., Shiers, H.M., Brown, G.W. \& Herbert, J. (2000). Morning cortisol as a risk factor for subsequent major depressive disorder in adult women. British Journal of Psychiatry 177: 505-510.

Heuser, I., Yassouridis, A. \& Holsboer, F. (1994). The combined dexamethasone CRH test - A refined laboratory test for psychiatric disorders. Journal of Psychiatric Research 28 (4): 341-356.

Kagan, J. (1994). Galen’s Prophecy. Basic Books, New York.

Kagan, J., Reznick, J.S. \& Snidman, N. (1987). The Physiology and Psychology of BehavioralInhibition in Children. Child Development 58 (6): 1459-1473. 
Kessler, R.C., McGonagle, K.A., Zhao, S.Y., Nelson, C.B., Hughes, M., Eshleman, S., Wittchen, H.U. \& Kendler, K.S. (1994). Lifetime and 12-month prevalence of DSM-III-R Psychiatric-Disorders in the United States- Results from the National-Comorbidity Study. Archives of General Psychiatry 51 (1): 8-19.

Kessler, R.C., Stein, M.B. \& Berglund, P. (1998). Social phobia subtypes in the national comorbidity study. American Journal of Psychiatry 155 (5): 613-619.

Maier, W., Lichtermann, D., Minges, J., Oehrlein, A. \& Franke, P. (1993). A controlled family study in panic disorder. Journal of Psychiatric Research 27: 79-87.

Mantella, R.C., Butters, M.A., Amico, J.A., Mazumdar, S., Rollman, B.L., Begley, A.E., Reynolds, C.F. \& Lenze, E.J. (2008). Salivary cortisol is associated with diagnosis and severity of late-life generalized anxiety disorder. Psychoneuroendocrinology 33 (6): 773-781.

Mattick, R.P. \& Clark, J.C. (1998). Development and validation of measures of social phobia scrutiny fear and social interaction anxiety. Behavior Research and Therapy 36 (4): 455-470.

Merikangas, K.R., Lieb, R., Wittchen, H.U. \& Avenevoli, S. (2003). Family and high-risk studies of social anxiety disorder. ACTA Psychiatrica Scandinavica 108: 28-37.

Meyer, T.J., Miller, M.L., Metzger, R.L. \& Borkovec, T.D. (1990). Development and validation of the Penn State Worry Questionnaire. Behaviour Research and Therapy 28 (6): 487-495.

Murray, L., Cooper, P., Creswell, C., Schofield, E. \& Sack, C. (2007). The effects of maternal social phobia on mother-infant interactions and infant social responsiveness. Journal of child psychology and psychiatry 48 (1): 45-52.

Murray, L., Creswell, C. \& Cooper, P. (2009). The development of anxiety disorders in childhood: an integrative review. Psychological Medicine 39 (9): 1413-1423.

Murray, L., de Rosnay, M., Pearson, J., Bergeron, C., Schofield, E., Royal-Lawson, M. \& Cooper, P. (2008). Intergenerational transmission of social anxiety: The role of social referencing processes in infancy. Child Development 79 (4): 1049-1064. 
Murray, L., Halligan, S.L., Goodyer, I. \& Herbert, J. (2010). Disturbances in early parenting of depressed mothers and cortisol secretion in offspring: A preliminary study. Journal of Affective Disorders 122 (3): 218-223.

Nelson, E.C., Grant, J.D., Bucholz, K.K., Glowinski, A., Madden, P.A.F., Reich, W. \& Heath, A.C. (2000). Social phobia in a population-based female adolescent twin sample: co-morbidity and associated suicide-related symptoms. Psychological Medicine 30 (4): 797-804.

Plotsky, P.M. (1991). Pathways to the secretion of adrenocorticotropin: A view from the portal. Journal of Neuroendocrinology 3 (1): 1-9.

Roelofs, K., van Peer, J., Berretty, E., de Jong, P., Spinhoven, P. \& Elzinga, B.M. (2008). Hypothalamus-Pituitary-Adrenal Axis Hyperresponsiveness is Associated with Increased Social Avoidance Behavior in Social Phobia. Biological Psychiatry, In Press.

Rosmalen, J.G.M., Oldehinkel, A.J., Ormel, J, de Winter, A.F., Buitelaar, J.K. \& Verhulst, F.C. (2005). Determinants of salivary cortisol levels in 10-12 year old children; a population-based study of individual differences. Psychoneuroendocrinology 30: 483-495.

Rubin, R.T., Phillips, J.J., Sadow, T.F. \& McCracken, J.T. (1995). Adrenal-gland volume in major depression- increase during the depressive episode and decrease with successful treatment. Archives of General Psychiatry 52 (3): 213-218.

Schmidt, L.A., Fox, N.A., Rubin, K.H., Sternberg, E.M., Gold, P.W., Smith, C.C. \& Schulkin, J. (1997). Behvioral and Neuroendocrine Responses in Shy Children. Developmental Psychobiology 30 (2): 127-140.

Schneier, F.R., Johnson, J., Hornig, C.D., Liebowitz, M.R. \& Weissman, M.M. (1992). Social Phobiacomorbidity and morbidity in an epidemiologic sample. Archives of General Psychiatry 49 (4): $282-288$.

Selye, H. (1973). Evolution of stress concept. American Scientist 61 (6): 692-699.

Schiefelbein, V.L. \& Susman, E.J. (2006). Cortisol levels and longitudial cortisol change as predictors of anxiety in adolescents. Journal of Early Adolescence 26 (4): 397-413. 
Schwartz, E.B., Granger, D.A., Susman, E.J., Gunnar, M.R. \& Laird, B. (1998). Assessing salivary cortisol in studies of child development. Child Development 69 (6): 1503-1513.

Smider, N.A., Essex, M.J., Kalin, N.H., Buss, K.A., Klein, M.H., Davidson, R.J. \& Goldsmith, H.H. (2002). Salivary Cortisol as a Predictor of Socioemotional Adjustment during Kindergarten: A Prospective Study. Child Development 73 (1): 75-92.

Stein, M.B., Chartier, M.J., Hazen, A.L., Kozak, M.V., Tancer, M.E., Lander, S., Furer, P., Chubaty, D. \& Walker, J.R. (1998). A direct-interview family study of generalized social phobia. American Journal of Psychiatry 155 (1): 90-97.

Sumner, M.M., Bernard, K. \& Dozier, M. (2010). Young Children's Full-Day Patterns of Cortisol Production on Child Care Days. Archives of Pediatrics and Adolescent Medicine 164 (6): $567-$ 571.

Tout, K., de Haan, M., Campbell, E.K. \& Gunnar, M.R. (1998). Social behavior correlates of cortisol activity in child care: Gender differences and time-of-day effects. Child Development 69 (5): $1247-1262$.

Turner-Cobb, J.A., Rixon, L. \& Jessop, D.S. (2008). A prospective study of diurnal cortisol responses to the social experience of school transition in four-year-old children: Anticipation, exposure, and adaptation. Developmental Psychobiology 50 (4): 377-389.

Uhde, T.W., Tancer, M.E., Gelernter, C.S. \& Vittone, B.J. (1994). Normal urinary free cortisol and post-dexamethasone cortisol in social phobia- comparison to normal volunteers. Journal of Affective Disorders 30 (3): 155-161.

Verhulst, F.C., vanderEnde, J., Ferdinand, R.F. \& Kasius, M.C. (1997). The prevalence of DSM-III-R diagnoses in a national sample of Dutch adolescents. Archives of General Psychiatry 54 (4): 329 336.

Warren, S.L., Gunar, M.R., Kagan, J., Anders, T.F., Simmens, S.J., Rones, M., Wease, S., Aron, E., Dahl, R.E. \& Sroufe, A. (2003). Maternal Panic Disorder: Infant Temperament, Neurophysiology, and Parenting Behaviors. Journal of the American Academy of Child and Adolescent Psychiatry 42 (7): 814-825. 
Watamura, S.E., Donzella, B., Alwin, J. \& Gunnar, M.R. (2003). Morning-to-afternoon increases in cortisol concentrations for infants and toddlers at child care: Age differences and behavioral correlates. Child Development 74 (4): 1006-1020.

Wedekind, D., Bandelow, B., Broocks, A., Hajak, G. \& Ruther, E. (2000). Salivary, total plasma and plasma free cortisol in panic disorder. Journal of Neural Transmission 107 (7): 831-837. 
Table 1. Characteristics of the sample: descriptive statistics

\begin{tabular}{|c|c|c|c|}
\hline Maternal Characteristics & Social Phobia & Comparison & GAD \\
\hline Age at recruitment (years): Mean (SD) & $30.01(4.9)$ & $31.2(4.2)$ & $30.7(3.6)$ \\
\hline Ethnicity- Caucasian: $\mathrm{N}(\%)$ & $67(100)$ & $89(94.7)$ & $48(84.7)$ \\
\hline Married/cohabiting: $\mathrm{N}(\%)$ & $63(94)$ & $90(95.7)$ & $54(96.4)$ \\
\hline Low SES: & $9(14.3)$ & $7(7.4)$ & $3(5.4)$ \\
\hline Child Characteristics & Social Phobia & Comparison & GAD \\
\hline Sex: Male & $25(37.3)$ & $47(50)$ & $26(46.4)$ \\
\hline First born: & $48(71.6)$ & $76(80.9)$ & $39(69.6)$ \\
\hline Birth Weight (gm): Mean (SD) & $3552(431)$ & $3506(488)$ & $3483(507)$ \\
\hline Age in months at BI assessment: Mean (SD) & $14.33(.7)$ & $14.36(.7)$ & $14.25(.7)$ \\
\hline Age in years when starting school: Mean (SD) & $4.65(.41)$ & $4.74(.22)$ & $4.71(.26)$ \\
\hline
\end{tabular}


Table 2. Mean cortisol levels and sampling times by maternal group at each time-block-unadjusted descriptive statistics

\begin{tabular}{|c|c|c|c|c|c|c|c|c|c|}
\hline & \multicolumn{3}{|c|}{ Before starting school } & \multicolumn{3}{|c|}{ First week of school } & \multicolumn{3}{|c|}{ End of term } \\
\hline & SP & $\mathbf{C}$ & GAD & SP & $\mathbf{C}$ & GAD & SP & $\mathbf{C}$ & GAD \\
\hline Morning & $(n=30)$ & $(\mathrm{n}=35)$ & $(n=23)$ & $(n=30)$ & $(n=35)$ & $(n=23)$ & $(n=30)$ & $(\mathrm{n}=35)$ & $(\mathrm{n}=23)$ \\
\hline Mean ng/ml (sd) & $2.93(1.5)$ & $3.36(1.6)$ & $2.81(1.4)$ & $3.24(1)$ & $3.51(1.5)$ & $3.19(1.4)$ & $3.4(1.5)$ & $3.32(1.2)$ & $2.95(.9)$ \\
\hline $\begin{array}{l}\text { Mean time } \\
\text { of spit }^{\text {a }}(\mathrm{sd})\end{array}$ & $7.9(.7)$ & $7.9(.8)$ & $7.8(.6)$ & $7.4(.3)$ & $7.5(.4)$ & $7.5(.4)$ & $7.4(.4)$ & $7.4(.4)$ & $7.5(.5)$ \\
\hline $\begin{array}{l}\text { Mean time from } \\
\text { waking in } \operatorname{mins}^{\mathrm{b}}(\mathrm{sd})\end{array}$ & $31.7(16)$ & 27 (15.6) & $28.8(16.2)$ & $22.2(9.3)$ & $\begin{array}{l}27.6 \\
(12.1)\end{array}$ & $27(9.7)$ & $\begin{array}{l}27.7 \\
(15.3)\end{array}$ & $\begin{array}{l}28.8 \\
(14.4)\end{array}$ & $26.5(13.4)$ \\
\hline Afternoon & $(\mathrm{n}=26)$ & $(n=33)$ & $(\mathrm{n}=21)$ & $(n=26)$ & $(n=33)$ & $(\mathrm{n}=21)$ & $(n=26)$ & $(\mathrm{n}=33)$ & $(n=21)$ \\
\hline Mean ng/ml (sd) & $.89(.6)$ & $.72(.6)$ & $.68(.5)$ & $.87(.8)$ & $.88(.9)$ & $.7(.3)$ & $.92(.5)$ & $.82(.5)$ & $.93(.4)$ \\
\hline Mean time of spit ${ }^{\mathrm{a}}$ (sd) & $16.5(.5)$ & $16.4(.6)$ & $16.6(.7)$ & $16.4(.3)$ & $16.4(.5)$ & $16.4(.5)$ & $16.3(.7)$ & $16.3(.5)$ & $16.4(.6)$ \\
\hline Bedtime & $(\mathrm{n}=26)$ & $(n=29)$ & $(n=21)$ & $(n=26)$ & $(n=29)$ & $(\mathrm{n}=21)$ & $(n=26)$ & $(\mathrm{n}=29)$ & $(n=21)$ \\
\hline Mean ng/ml (sd) & $.28(.3)$ & $.56(.7)$ & $.55(.8)$ & $.55(.5)$ & $.48(.5)$ & $.41(.2)$ & $.56(.5)$ & $.53(.6)$ & $.35(.2)$ \\
\hline Mean time of spit ${ }^{\mathrm{a}}$ (sd) & $19.5(.6)$ & $19.5(.8)$ & $19.4(.6)$ & $19.3(.4)$ & $19.4(.5)$ & $19.5(.5)$ & $19.2(.4)$ & $19.5(.6)$ & $19.4(.4)$ \\
\hline
\end{tabular}

Note. ${ }^{\mathrm{a}}=$ minutes expressed as proportion of an hour, ${ }^{\mathrm{b}}=$ data available for a limited sample as mentioned in method section. $\mathrm{SP}=$ social phobia group. $\mathrm{C}=$ comparison group. $\mathrm{GAD}=$ generalised anxiety disorder group. 
Figure 1. Mean log-transformed morning cortisol levels across time-block, by maternal group.

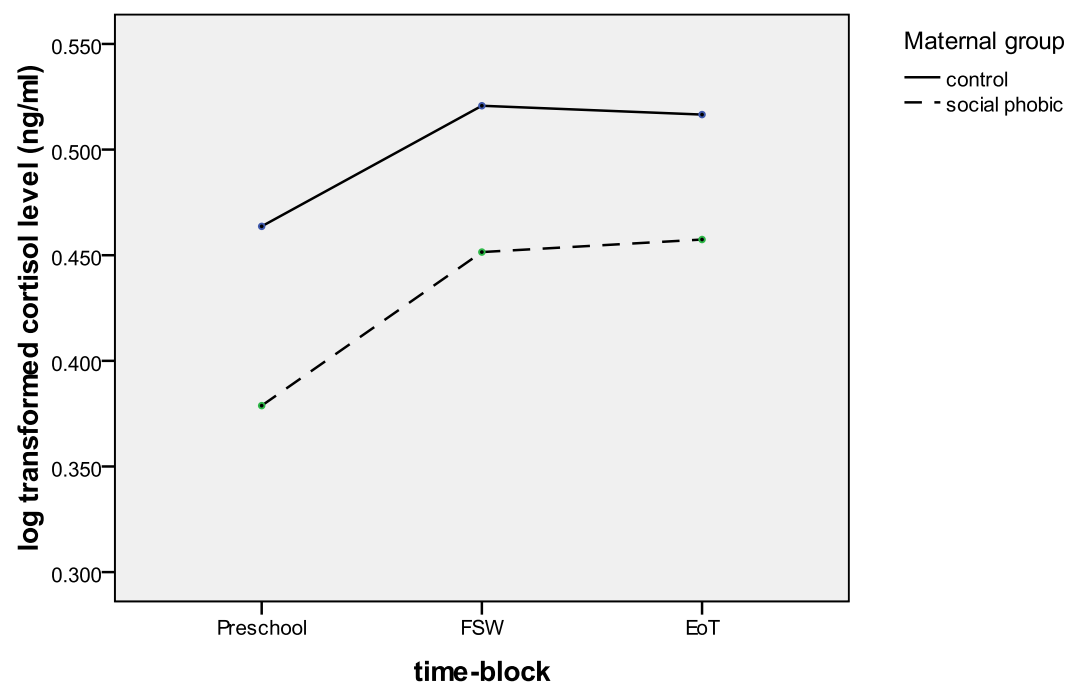

Note. Covariates appearing in the model are: child parity, the total number of cortisol values available at the FSW timeblock, mean difference in sampling time between BSS and FSW samples, and attendance at nursery or preschool.

Figure.2 Mean log-transformed afternoon cortisol levels across time-block, by maternal group

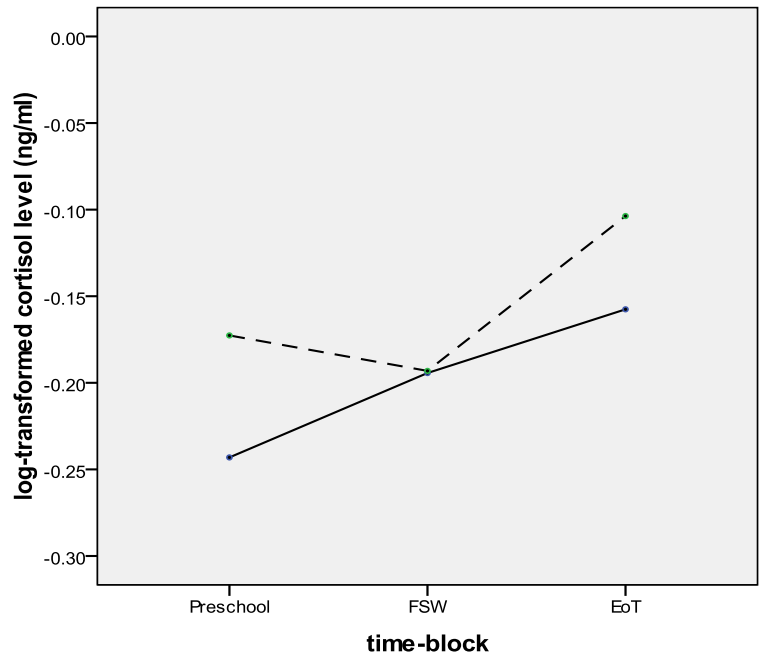

Maternal group

- control

- - social phobic

Note. Covariates appearing in the model are: child parity and the mean time of FSW samples 
Figure 3. Mean log-transformed bedtime cortisol levels across time-block, by maternal group

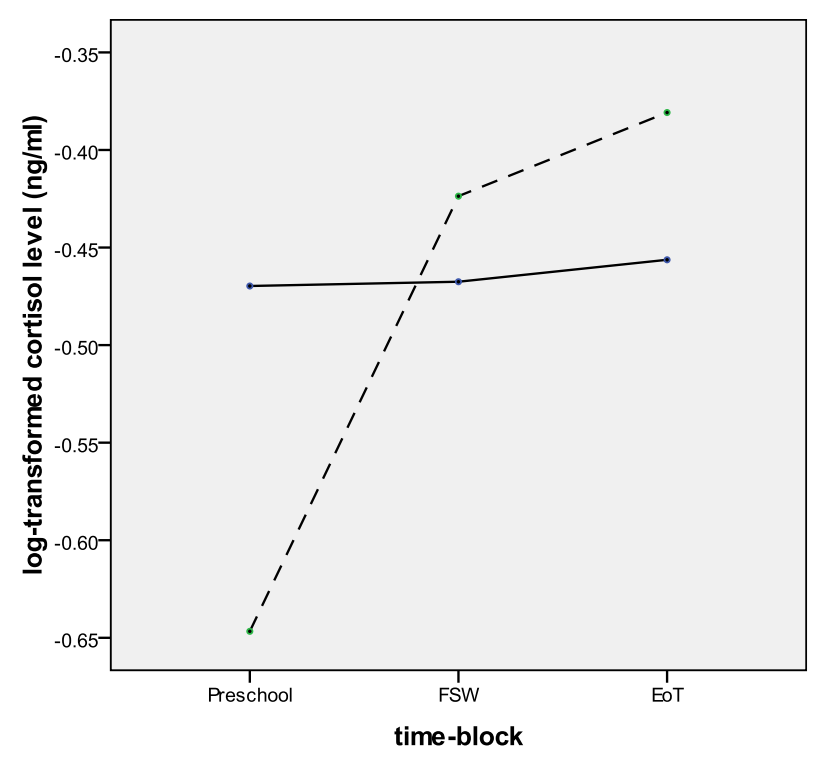

Maternal group

- control

Note. Covariates appearing in the model are: child parity.

Figure 4. Mean morning cortisol levels, by sex

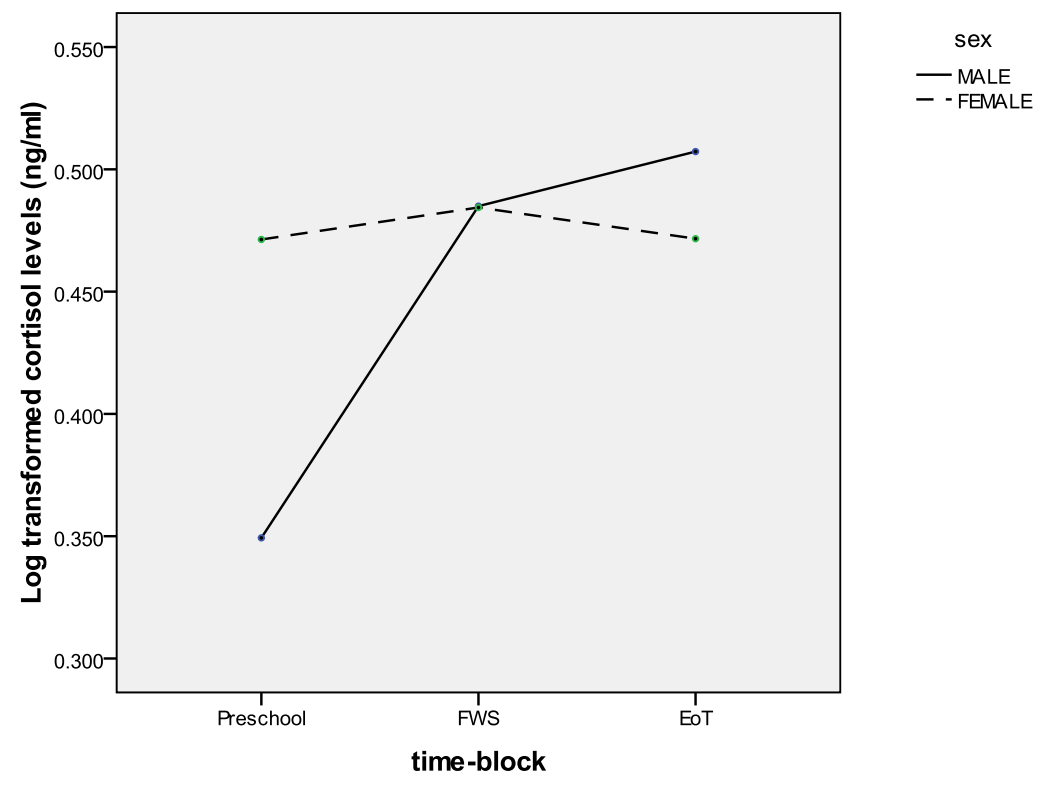


Figure 5. Mean 4pm cortisol levels, by child BI at 14 months

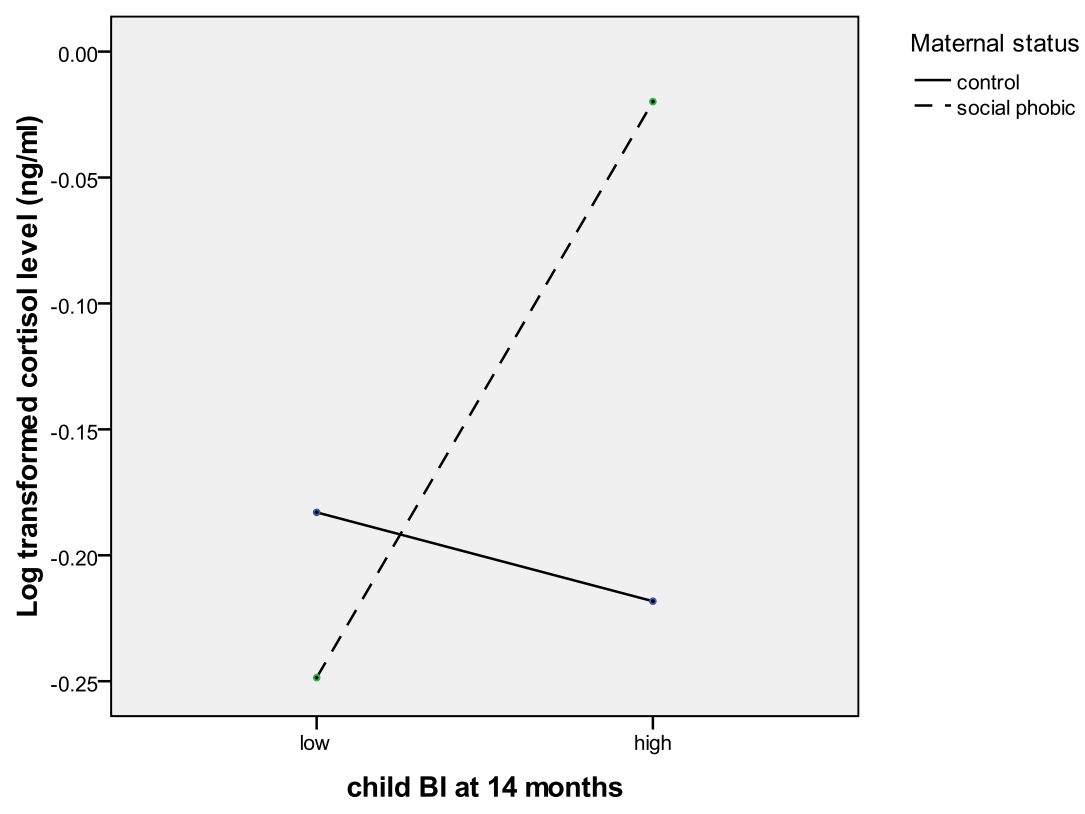


${ }^{*}$ Conflict of Interest

Conflict of Interest.

None of the authors has any conflict of interest. 
${ }^{*}$ Acknowledgement

Acknowledgements.

We thank Adriane Arteche for statistical advice. 


\section{${ }^{*}$ Contributors}

Contributors.

Lynne Murray, Peter Cooper, Joe Herbert, lan Goodyer and Megan Gunnar designed the study and wrote the protocol. Joe Herbert oversaw the analyses of salivary cortisol. Stephanie Russ collected data, undertook the statistical analyses and wrote the first draft of the manuscript. Tim Croudace and Megan Gunnar advised on the statistical analyses. Lynne Murray and Peter Cooper oversaw all aspects of the study and supervised Steph Russ; Lynne Murray made a substantial contribution to writing the paper. All authors contributed to and have approved the final manuscript. 
Role of Funding Source.

Funding for this study was provided by MRC (UK) Grant G0600990; and a studentship to S Russ from the ESRC (UK). The MRC and ESRC had no further role in study design; in the collection, analysis and interpretation of data; in the writing of the report; and in the decision to submit the paper for publication. 
Suggested Reviewers List:

1. Professor Vivette Glover - Department of Surgery and Cancer, Imperial College, London. Email: v.glover@imperial.ac.uk

2. Professor Thomas G O'Connor - University of Rochester, School of Medicine and Dentistry

3. Dr Stephen J Suomi, National Institute of Health. Email: suomis@mail.nih.gov

4. Dr Jacqueline Bruce. Oregon Social Learning Centre. 